\title{
Efecto de Butil Hidroxitolueno (BHT) en la Estabilidad Oxidativa de un Lubricante a Base de Aceite de Ajonjolí
}

\author{
Arnoldo E. Delgado ${ }^{(1,2) \star}$, Oscar Palacio ${ }^{(2)}$ y William Aperador ${ }^{(2)}$ \\ (1) Escuela Colombiana de Ingeniería Julio Garavito, Facultad de Ingeniería, Programa de Ingeniería \\ Industrial, Carrera 45 No. 205-59, Bogotá-Colombia. \\ (2) Universidad Militar Nueva Granada, Facultad de Ingeniería, Programa de Ingeniería Mecatrónica, \\ Carrera 11 No.101-80, Bogotá-Colombia. (e-mail: articulopublish@gmail.com)
}

${ }^{*}$ Autor a quien debe dirigirse la correspondencia

Recibido Oct. 30, 2014; Aceptado Dic. 23, 2014; Versión final Feb. 13, 2015, Publicado Ago. 2015

\begin{abstract}
Resumen
En este estudio se analizó el efecto del antioxidante butil hidroxitolueno sobre la degradación oxidativa del aceite de ajonjolí (Sesamum indicum). Se evaluaron diferentes concentraciones de antioxidante (0.05; 0.1; 0.2 ; y $0.5 \% \mathrm{p} / \mathrm{p}$ ). La estabilidad oxidativa fue determinada mediante análisis de calorimetría diferencial de barrido, El aceite con $0.1 \%$ de antioxidante presentó mayor resistencia a la oxidación. Varias muestras de aceite fueron preparadas con esta concentración para evaluar el efecto del antioxidante en otras propiedades. Un muestra con la misma cantidad de butil hidroxitolueno mostró menor degradación del aceite de ajonjolí sometido a la prueba tribológica de desgaste preventivo sin modificación del poder lubricante. La adición de butil hidroxitolueno en la mencionada cantidad no modificó el índice de viscosidad del aceite. Sin embargo se observó ligeros cambios en la temperatura de cristalización y fusión del aceite de ajonjolí.
\end{abstract}

Palabras claves: aceite de ajonjolí, BHT, DSC, estabilidad oxidativa, tribología

\section{Effect of Butylated Hydroxytoluene (BHT) on the Oxidative Stability of a Lubricant Based on Sesame Oil}

\begin{abstract}
In this study, the effects of butylated hydroxytoluene on degradation characteristics of sesame oil (Sesamum indicum) were investigated using different concentrations of antioxidant $(0.05 ; 0.1 ; 0.2$ and 0.5 $\% \mathrm{w} / \mathrm{w})$. Oxidative stability of samples was determined by differential scanning calorimetry. Adding $0.1 \%$ antioxidant showed higher oxidation resistance of sesame oil. Various samples with concentration of $0.1 \%$ antioxidant were prepared in order to analyze the effect of BHT on other properties of the oil. A sample with the same amount of butylated hydroxytoluene showed less degradation of sesame oil in tribological testing (Preventive Wear) without altering the lubricity. Adding the same amount of antioxidant did not modify the viscosity index, but affected the melting and crystallization temperature of sesame oil
\end{abstract}

Keywords: sesame oil, BHT, DSC, oxidative stability, tribology 


\section{INTRODUCCIÓN}

Los aceites vegetales tienen excelentes propiedades como base para el desarrollo de lubricantes, en particular alto índice de viscosidad, buena lubricidad, baja volatilidad y especialmente, son renovables, biodegradables y no tóxicos; sin embargo, existen algunas limitaciones que deben ser superadas, tales como la cristalización a bajas temperaturas, la baja estabilidad térmica, oxidativa e hidrolítica (Quinchia et al., 2012). El aceite de ajonjolí tiene ventajas con respecto a otros aceites vegetales debido a que contiene lignanos (sesamina y sesamolina), compuestos que contribuyen a una mayor estabilidad oxidativa (Sathwik et al., 2012; Hemalatha y Ghafoorunissa, 2007); sin embargo, para su desempeño como lubricante se requiere la incorporación de antioxidantes y aditivos antidesgaste, que retrasen significativamente el proceso de auto-oxidación, la degradación del aceite por fricción y que mejoren el poder lubricante.

Los aceites vegetales poseen alto contenido de ácidos grasos insaturados; generalmente, los porcentajes de ácidos grasos encontrados en diferentes aceites vegetales cumplen con un 80,17-82,91\% de ácidos grasos insaturados y 15,38 - 17,25\% de ácidos grasos saturados (Lafont et al., 2014; San José et. al., 2014; Hernández et al., 2007). El alto porcentaje de ácidos grasos insaturados disminuye la estabilidad oxidativa, mientras que una mayor proporción de ácidos grasos saturados de cadena larga conduce a una disminución de la fluidez en frío (Delgado y Aperador, 2014).

El mecanismo de oxidación típico de un aceite vegetal se origina con la reacción de iniciación (1), el oxígeno ataca el doble enlace de los ácidos grasos insaturados formando un radical libre $R^{*}$. En las etapas de propagación el radical $\mathrm{R}^{*}$ reacciona con el oxígeno para formar radical peroxi $\mathrm{ROO}$ * (2). El radical $\mathrm{ROO}^{*}$ ataca otra molécula lipídica $\mathrm{RH}$ dando origen a un hidroperóxido $\mathrm{ROOOH}$ y otro radical libre $\mathrm{R}^{\star}$ extendiendo así el proceso de oxidación (3) y (4). Los hidroperóxidos se acumulan en el aceite y también se descomponen en compuestos secundarios por ejemplo epóxidos, esta etapa se denomina terminación (5) y (6).

$\mathrm{RH}$

$\underset{\mathrm{O}_{2} \text { (presencia de catalizador) }}{\longrightarrow} \mathrm{R}^{\bullet}+\mathrm{H}^{\bullet}$

$\mathrm{R}^{\bullet}+\mathrm{O}_{2} \underset{\mathrm{O}_{2} \text { (presencia de catalizador) }}{\longrightarrow} \mathrm{ROO}$

$\mathrm{ROO}^{\circ}+\mathrm{RH} \underset{\mathrm{O}_{2} \text { (presencia de catalizador) }}{\longrightarrow} \mathrm{ROOH}+\mathrm{R}^{\circ}$

$\mathrm{ROO}^{\circ}+\mathrm{ROO}^{\circ} \underset{\mathrm{O}_{2} \text { (presencia de catalizador) }}{\longrightarrow} \mathrm{ROOR}+\mathrm{O}_{2}$

$\mathrm{ROO}^{\circ}+\mathrm{R}^{\bullet}$

$\underset{\mathrm{O}_{2} \text { (presencia de catalizador) }}{\longrightarrow} \mathrm{ROOR}$

$\mathrm{R}^{\bullet}+\mathrm{R}^{\bullet}$

$\underset{\mathrm{O}_{2} \text { (presencia de catalizador) }}{\longrightarrow} 2 \mathrm{R}$

La oxidación de los aceites vegetales se puede controlar o retardar usando adecuadamente antioxidantes. Éstos se clasifican en primarios y secundarios según su modo de acción. Los de tipo primario o interruptores de cadena, actúan en la etapa de propagación reaccionando con los radicales para convertirlos en compuestos más estables; los antioxidantes secundarios, reducen la velocidad de la etapa de iniciación de formación de radicales libres. En aceites vegetales, los antioxidantes más empleados son los terminadores de radicales libres tales como butilhidroxianisol (BHA), butilhidroxitolueno $(\mathrm{BHT})$ o terbutil dihidroquinona (TBHQ) (Rizwanul et al., 2014; Fox y Stachowiak, 2007; Rojano, 1997); dichos antioxidantes, son efectivos a bajas concentraciones y algunos se pueden convertir en pro-oxidantes a altas concentraciones (Navas, 2005). El BHT es soluble en aceites e insoluble en agua, se usa para preservar aceites vegetales y es considerado como el antioxidante más ampliamente usado en lubricantes de origen vegetal y productos alimenticios, tales como cereales, grasas comestibles, arroz y productos de confitería (Tuner y Korkmaz, 2007; Igoe, 2011; Marteau et al., 2014). La oxidación de un aceite vegetal es una reacción exotérmica, el cambio en la entalpía puede ser medido con la técnica de calorimetría diferencial de barrido (DSC). Los parámetros a evaluar son la temperatura de comienzo de la oxidación (TONSET) y el tiempo de inducción a la oxidación (To) (Tan et al., 2002; Pardauil et al., 2011).

En esta investigación se analizó el poder antioxidante del BHT en el aceite de ajonjolí, se evaluaron cuatro (4) concentraciones empleando la técnica de estabilidad oxidativa por medio de DSC. La mezcla de aceite y 
antioxidante que arrojó mayor resistencia a la oxidación fue elegida para realizar los siguientes ensayos: 1) Prueba tribológica de Desgaste Preventivo: se analizó el efecto del BHT sobre el poder lubricante y la degradación del aceite usado en la prueba por medio de DSC y Valor de Peróxidos. 2) Simultáneamente se prepararon muestras de aceite con similar concentración de BHT para analizar su efecto sobre el índice de viscosidad y el comportamiento a bajas temperaturas (picos de cristalización y fusión) mediante DSC.

\section{MATERIALES Y MÉTODOS}

\section{Materiales}

Aceite de ajonjolí extra virgen, prensado en frio, densidad: $0,98 \mathrm{~g} / \mathrm{cm}^{3}$, acidez: $0,33 \mathrm{mg}-\mathrm{KOH} / \mathrm{g}$, viscosidad cinemática: 34,64 cSt, Lote 077052014C, comercializado por BIO-ESSENS ${ }^{\circledR}$. Antioxidante: BHT, densidad: $1,048 \mathrm{~g} / \mathrm{cm}^{3}$, punto de fusión: $70^{\circ} \mathrm{C}$.

\section{Perfil de ácidos grasos}

El perfil de ácidos grasos se realizó siguiendo el método FAMEs AOAC 965.49 modificado, con una columna Rtx ${ }^{\circledR}-$ Wax $30 \mathrm{~m} ; 0,25 \mathrm{~mm}$ ID; 0,5um, con las siguientes especificaciones para el programa: 100 ${ }^{\circ} \mathrm{C} / 2 \mathrm{~min}, 250{ }^{\circ} \mathrm{C} / 11 \mathrm{~min}\left(21^{\circ} \mathrm{C} / \mathrm{min}\right)$ volumen de inyección $4 \mu \mathrm{L}$. Equipo: cromatógrafo de gases serie 101652, modelo Varian ${ }^{\circledast} 3900$, clase FID (Flame Ionization Detector).

\section{Análisis DSC}

El instrumento, DSC Q20 Serie: S/N RCS 1-3378 de TA Instruments ${ }^{\circledR}$, se calibró con indio puro (punto de fusión $156,60^{\circ} \mathrm{C} ; \Delta \mathrm{Hf}=28,45 \mathrm{~J} / \mathrm{g}$ ). Las muestras se pesaron en crisoles de aluminio abiertos y se colocaron en la celda DSC Tzero $^{\circledR}$ con un crisol vacío como referencia.

En el ensayo de estabilidad oxidativa, las muestras se purgaron con un flujo de $50 \mathrm{~mL} / \mathrm{min}$ de aire y se llevaron desde $10^{\circ} \mathrm{C}$ hasta $250^{\circ} \mathrm{C}$ con una velocidad de calentamiento de $10^{\circ} \mathrm{C} / \mathrm{min}$. A partir de las curvas de calentamiento flujo de calor $(\mathrm{W} / \mathrm{g})$ en función de la temperatura, se determinaron las temperaturas de comienzo de la oxidación (TONSET). La evaluación de la estabilidad oxidativa se realizó con los tiempos de inducción a la oxidación ( $\left.\mathrm{T}_{0}\right)$, obtenidos por extrapolación a partir de las curvas DSC isotérmicas de oxidación flujo de calor $(\mathrm{W} / \mathrm{g})$ en función del tiempo. La temperatura isotérmica fue programada a $165^{\circ} \mathrm{C}$ y las muestras se oxidaron en un flujo de aire de $50 \mathrm{~mL} / \mathrm{min}$. Los datos se analizaron con el software Universal Analysis 2000, versión 4.5A (TA Instruments®).

Para la determinación de los máximos de cristalización y fusión, la muestra fue purgada con flujo de nitrógeno de $20 \mathrm{~mL} / \mathrm{min}$ y sometida a tres programas de temperatura: 1. Calentamiento desde la temperatura ambiente hasta $50^{\circ} \mathrm{C}$, la isoterma se mantuvo durante $5 \mathrm{~min}$. 2. Enfriamiento desde $50{ }^{\circ} \mathrm{C}$ hasta $-80^{\circ} \mathrm{C}$, isoterma durante $5 \mathrm{~min}$. 3. Calentamiento desde $-80^{\circ} \mathrm{C}$ hasta $50{ }^{\circ} \mathrm{C}$. La velocidad de calentamiento y enfriamiento fue $10^{\circ} \mathrm{C} / \mathrm{min}$.

\section{Valor de Peróxidos (VP)}

Se determinó de acuerdo con el método establecido por la Asociación de Comunidades Analíticas A.O.A.C. 965.33. Corresponde a la cantidad de sustancias en la muestra que oxidan el yoduro de potasio en las condiciones de prueba, se expresa en mili-equivalentes de peróxido por kilogramo (mEq peróxido/kg).

\section{Ensayo tribológico}

Se realizó el ensayo de Desgaste Preventivo (DP) en un tribómetro cuatro bolas, según la norma ASTM D4172-94. Se emplearon esferas de acero de aleación de cromo AISI 52100, con 12,7 mm de diámetro, dureza de 60-66 HRC. Condiciones de la prueba: temperatura $75^{\circ} \mathrm{C}$, velocidad $1200 \mathrm{rpm}$, carga 147 Newton, tiempo $60 \mathrm{~min}$. El coeficiente de fricción (f) y el diámetro promedio de huella (DPH) son los parámetros mediante los cuales se evaluó la lubricidad de la mezcla de aceite de ajonjolí con BHT; la medición de cada parámetro se realizó por triplicado.

\section{Índice de Viscosidad}

Determina la correlación existente entre la viscosidad de un aceite lubricante y la temperatura, se estableció según la norma ASTM D445-12 y mediante la ecuación (7). 
$I V=\left(\frac{L-U}{L-H}\right) * 100$

En esta ecuación., $L$ es la Viscosidad cinemática a $40{ }^{\circ} \mathrm{C}$ de un aceite de referencia que presenta gran variación de viscosidad con la temperatura $\left(\mathrm{m}^{2} / \mathrm{s}\right)$. Su índice de viscosidad se fija en $0 ; H$ es la Viscosidad cinemática a $40{ }^{\circ} \mathrm{C}$ de un aceite de referencia que presenta escasa variación de viscosidad con la temperatura $\left(\mathrm{m}^{2} / \mathrm{s}\right)$. Su índice de viscosidad se fija en 100; y $U$ es la Viscosidad cinemática a $40{ }^{\circ} \mathrm{C}$ del aceite en estudio $\left(\mathrm{m}^{2} / \mathrm{s}\right)$.

\section{RESULTADOS Y DISCUSIÓN}

\section{Perfil de ácidos grasos}

En la Tabla 1 se presenta el valor de los ácidos grasos del aceite de ajonjolí, en donde se observa que no hay gran diferencia entre la cantidad de cada uno de los ácidos comparándolos con la literatura. Sin embargo, se observa ligera variación en el porcentaje de los ácidos oleico y linoleico, Mannekote y Kalias en sus estudios presentan mayor proporción de ácido oleico y el resultado experimental arroja mayor cantidad de ácido linoléico. Los principales ácidos grasos de los aceites vegetales son los que poseen 18 carbonos, como oleico y linoleico lo que indica mejor fluidez a temperaturas bajas (Lafont et. al., 21014); así mismo, el aceite de ajonjolí contiene antioxidantes naturales tales como sesamina, sesamolina y sesamol, que lo protegen parcialmente de la oxidación (Sathwik et al., 2012).

Tabla 1: Composición de ácidos grasos del aceite de ajonjolí

\begin{tabular}{|l|c|c|}
\hline \multirow{2}{*}{ Nombre del ácido graso } & \multicolumn{2}{|c|}{ Fracción (\%) } \\
\cline { 2 - 3 } & $\begin{array}{c}\text { Sesamum indicum Oil } \\
\text { Bio Essens }^{\circledR}{ }^{\text {Lote 077052014C }}\end{array}$ & $\begin{array}{c}\text { Literatura } \\
\text { Mannekote y Kailas, 2012 }\end{array}$ \\
\hline Palmítico & 9,1 & 10,7 \\
\hline Palmitoleico & 0,15 & 0,2 \\
\hline Esteárico & 5,4 & 5,2 \\
\hline Oleico & 39,6 & 41,4 \\
\hline Linoléico & 44,6 & 40,4 \\
\hline Linolénico & 0,33 & 0,4 \\
\hline Araquídico & 0,64 & 0,4 \\
\hline Otros & 0,18 & 1,3 \\
\hline
\end{tabular}

\section{Concentración de BHT y estabilidad oxidativa}

La concentración del BHT influye en la estabilidad oxidativa del aceite vegetal. La temperatura de inicio de oxidación de cada una de las muestras se observa en la figura 1. El aceite con 0,1\% de BHT arrojó el valor más alto de TONSET $=193,46{ }^{\circ} \mathrm{C}$, concentraciones de antioxidante en el aceite superiores al valor mencionado disminuyen la temperatura de inicio de la oxidación, lo cual tiene efecto concordante con lo encontrado por Martínez e Iván (2013) quienes afirman que la eficiencia de todos los antioxidantes disminuye con el incremento de la concentración. La TONSET del aceite de ajonjolí sin antioxidante es de $189,02{ }^{\circ} \mathrm{C}$.

Adicionalmente, con los tiempos de inducción a la oxidación ( $\left.T_{0}\right)$, obtenidos a partir de las curvas DSC isotérmicas a $165^{\circ} \mathrm{C}$ (figura 2), se evaluó la estabilidad oxidativa de cada una de las muestras. El aceite de ajonjolí puro presenta un $\mathrm{T}_{0}$ de $36,31 \mathrm{~min}$, mientras que la mezcla con $0,1 \% \mathrm{BHT}$ es la que arroja un mayor To siendo éste de 40,42 min, resultados que concuerdan con los que se muestran en la figura 1.

\section{Incidencia del bht al 0,1\% en las propiedades lubricantes del aceite}

Los resultados se analizaron mediante ANOVA de una vía (figura 3) con el software Statgraphics® 5.0 Plus. La concentración de BHT al 0,1\% no influye significativamente en el coeficiente de fricción ni en el diámetro promedio de huella $(\alpha \geq 0,05)$. Según previa investigación, el aceite de ajonjolí sin aditivos, se degradó parcialmente cuando fue sometido a la prueba de desgaste preventivo (Delgado y Aperador, 2014). Con el fin de verificar si la concentración de $0,1 \%$ de BHT fue efectiva para disminuir la oxidación del aceite durante el ensayo tribológico, se determinó el valor de peróxidos y el tiempo de inducción a la oxidación del aceite usado. 


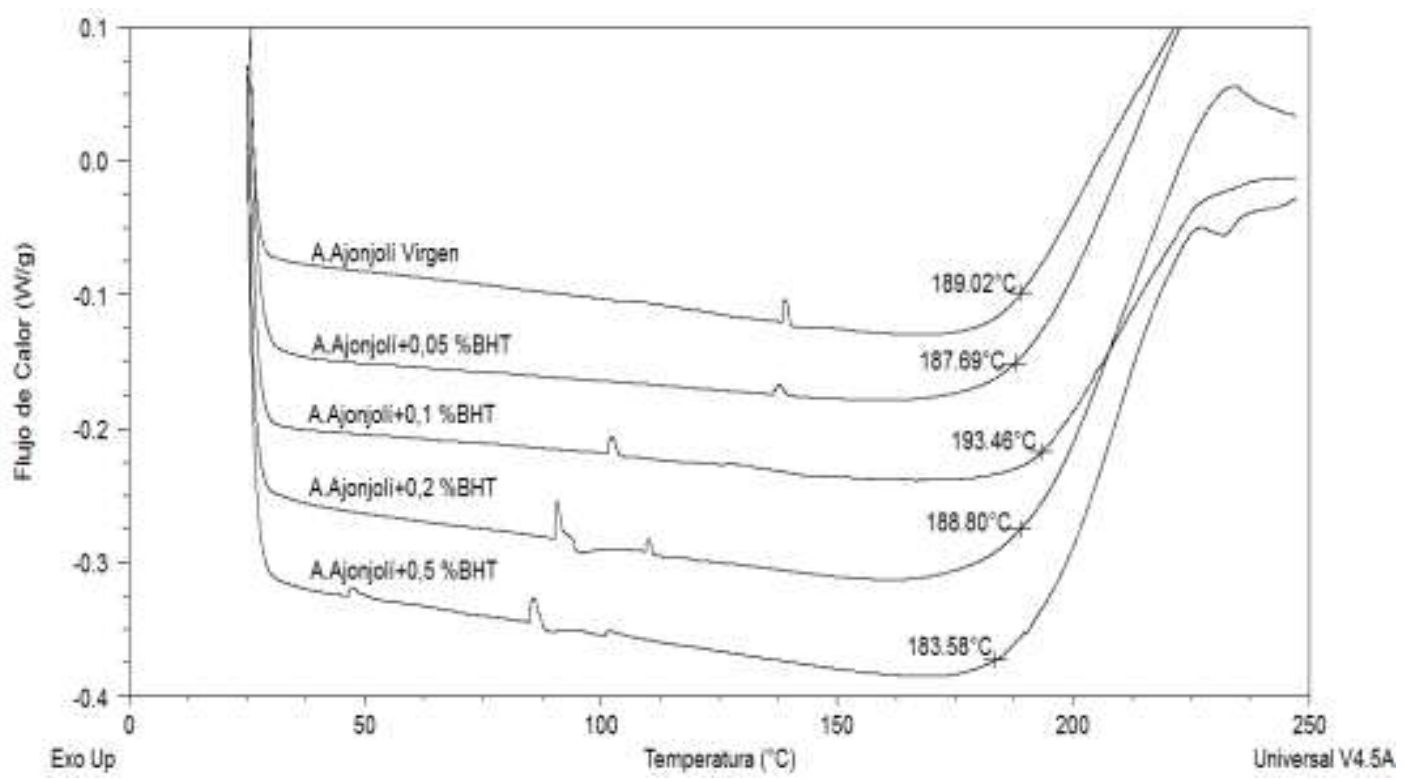

Fig. 1: Estabilidad oxidativa. Temperaturas ONSET

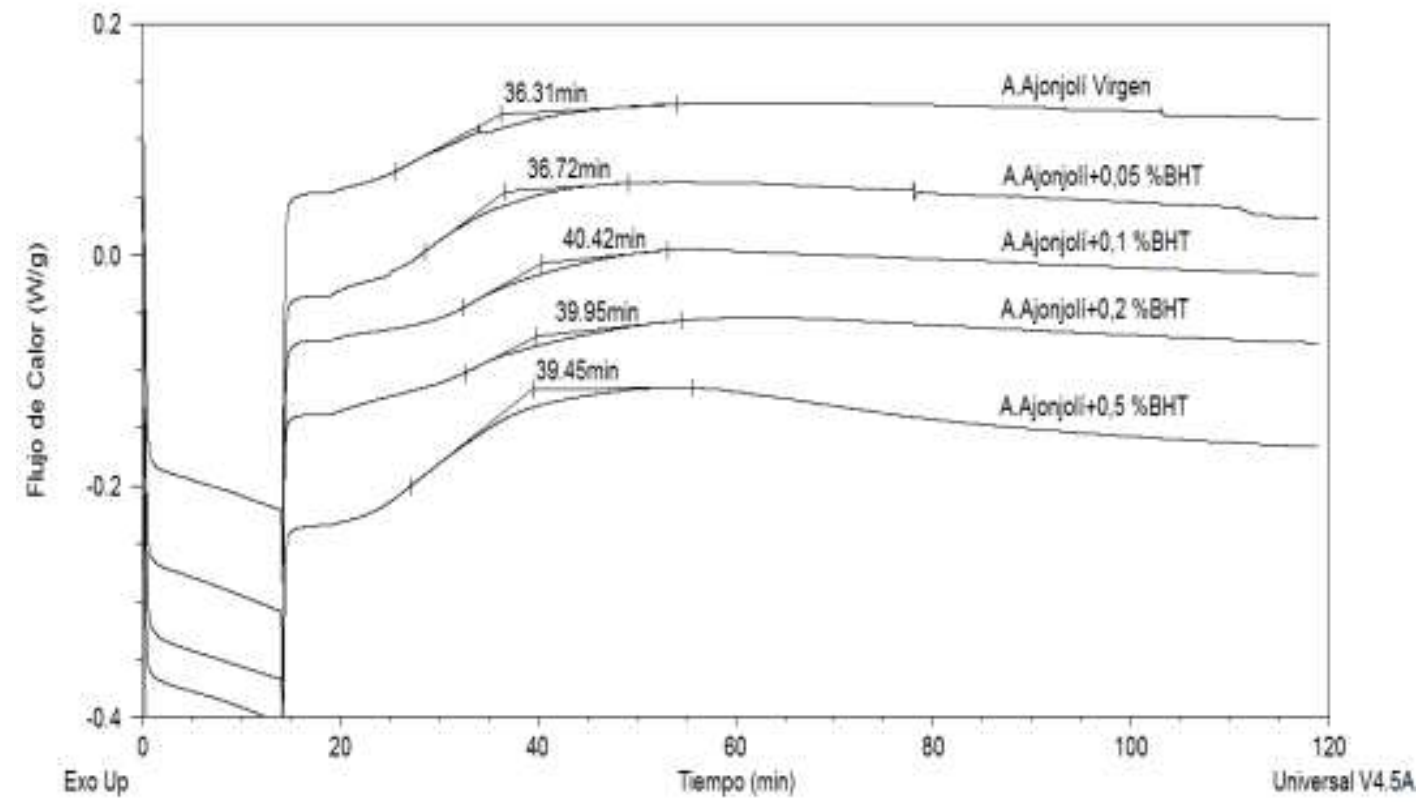

Fig. 2: Estabilidad oxidativa. Tiempos de inducción a $165^{\circ} \mathrm{C}$
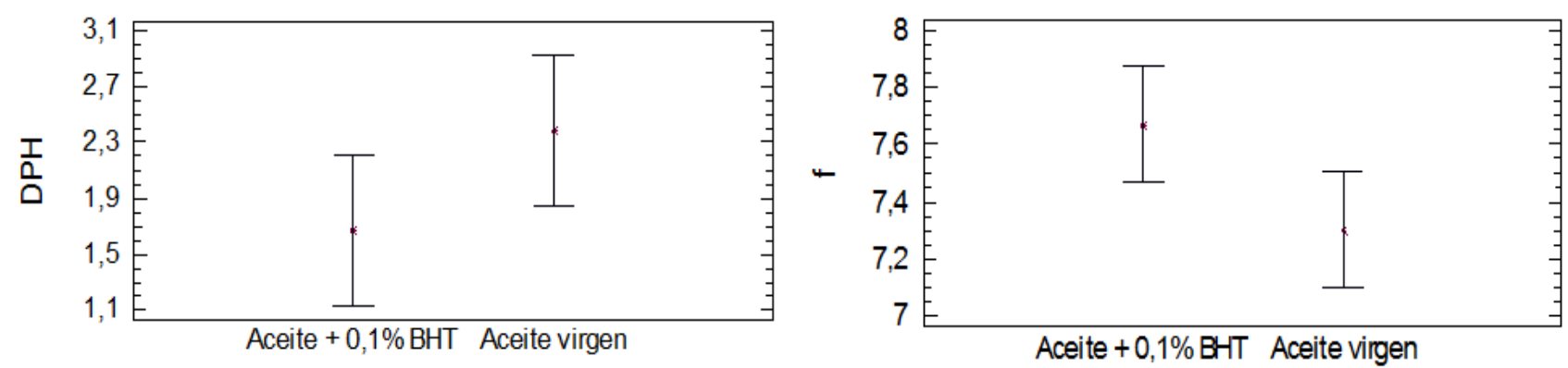

Fig. 3: Medias ANOVA de una vía - Diámetro promedio de huella (DHP) y coeficiente de fricción (f) 


\section{Valor de Peróxidos (VP)}

El BHT retarda el proceso de auto-oxidación del aceite vegetal; la mezcla de aceite de ajonjolí con antioxidante obtuvo un resultado de $10,92 \mathrm{mEq}$ peróxido/kg siendo éste un menor valor que el que presentó el aceite de ajonjolí DP sin aditivos (14,97 mEq peróxido/kg). El VP se redujo $27 \%$ debido al empleo de $\mathrm{BHT}$, el cual aumenta la estabilidad de la mezcla. Resultado acorde con otras investigaciones en dónde se evaluó el efecto del BHT en aceite de soya, canola, jatropha, girasol, palma y algodón siendo éste de gran aporte para el proceso de auto-oxidación, presenta mayor influencia que otros antioxidantes tales como terbutil-hidroquinona (TBHQ) y di-ter-butil-hidroquinona (DTBHQ) (Rizwanul et al., 2014).

\section{Tiempo de Inducción a la Oxidación}

El To para el aceite de ajonjolí sin aditivos fue de 21,01 min, mientras que el $\mathrm{T}_{0}$ del aceite con $\mathrm{BHT}$ al 0,1\% fue de 25,49 min, 4,48 unidades por encima (figura 4). Esto se debe a que el BHT, al ser un antioxidante primario, reacciona con los radicales libres para convertirlos en compuestos más estables, tanto a la temperatura como a la presencia de aire, minimizando así el proceso de auto-oxidación del aceite. De esta manera el antioxidante protegió al aceite de ajonjolí durante el ensayo tribológico DP.

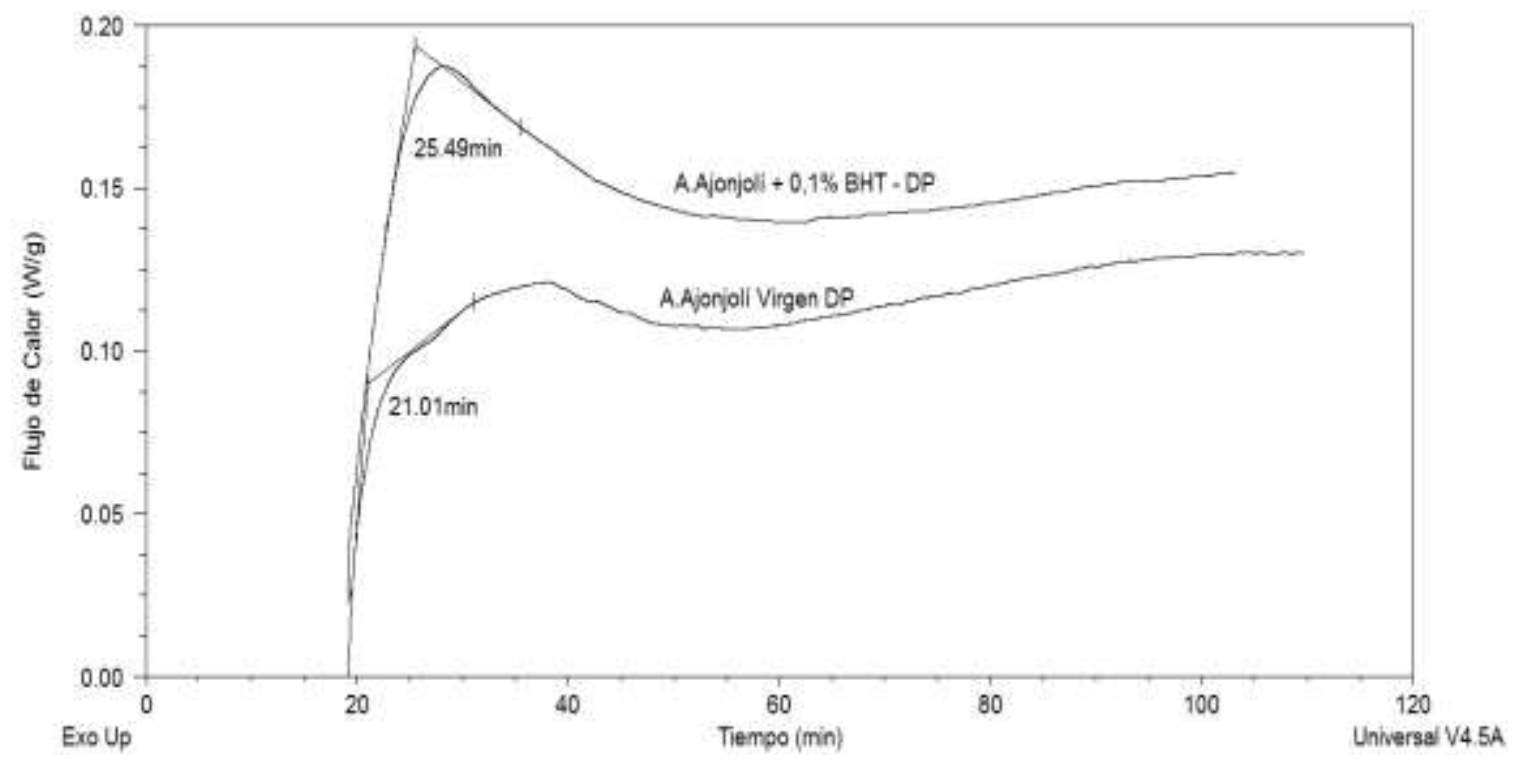

Fig. 4: Estabilidad oxidativa. Ensayo de desgaste preventivo

\section{ANÁLISIS DE ENFRIAMIENTO-CALENTAMIENTO}

Estudios anteriores han demostrado que la velocidad de enfriamiento y calentamiento influye en la forma de las curvas así como en la temperatura que se obtiene, la intensidad del pico de temperatura aumenta a medida que se incrementa la velocidad; adicional a esto, a mayor velocidad aparecen en las curvas DSC más manifestaciones endotérmicas y/o exotérmicas (Jiménez et al., 2007). Las condiciones a las que se llevó a cabo el análisis de las muestras en estudio (ver materiales y métodos) fueron tomadas de la investigación realizada por Adhvaryu et al. (2002).

En la curva de enfriamiento (figura 5) se observan dos picos exotérmicos en ambas muestras de aceite de ajonjolí (virgen y con $0,1 \%$ de BHT). Los picos más característicos en cada una de las curvas DSC de calentamiento son aquellos que tienen mayor magnitud (Tan y Che, 2000). En cristalización, frecuentemente se presentan dos picos exotérmicos, el primero corresponde a la temperatura de inicio de la formación de micro-cristales y el segundo a la temperatura de congelación de la muestra (Jiménez et al., 2007). Los picos son debidos a la presencia de diferentes polimorfismos de los triacilglicéridos, (formas a y $\beta$ ) que constituyen el aceite de ajonjolí (Quinchia et al., 2012). El aceite con 0,1\% de BHT es el primero en cristalizar a la temperatura de $-12,42^{\circ} \mathrm{C}(2,5$ grados antes que el aceite virgen) igual sucede con el segundo pico de cristalización cuyo valor es $-38,48^{\circ} \mathrm{C}$ (cristaliza $4,1^{\circ} \mathrm{C}$ antes que el aceite virgen).

En la curva de calentamiento (figura 6), se observan dos picos endotérmicos en cada uno de los aceites (virgen y con $0,1 \%$ de BHT). La curva de calentamiento guarda relación con la de enfriamiento, toda vez que el aceite virgen presentó el primer pico de fusión en $-32,83{ }^{\circ} \mathrm{C}\left(2,54{ }^{\circ} \mathrm{C}\right.$ antes que el aceite con $0,1 \%$ de $\mathrm{BHT}$ ), el segundo pico de fusión del aceite virgen fue $-20,07^{\circ} \mathrm{C}$ (fundió $3,84^{\circ} \mathrm{C}$ antes que el aceite que contiene el antioxidante). 


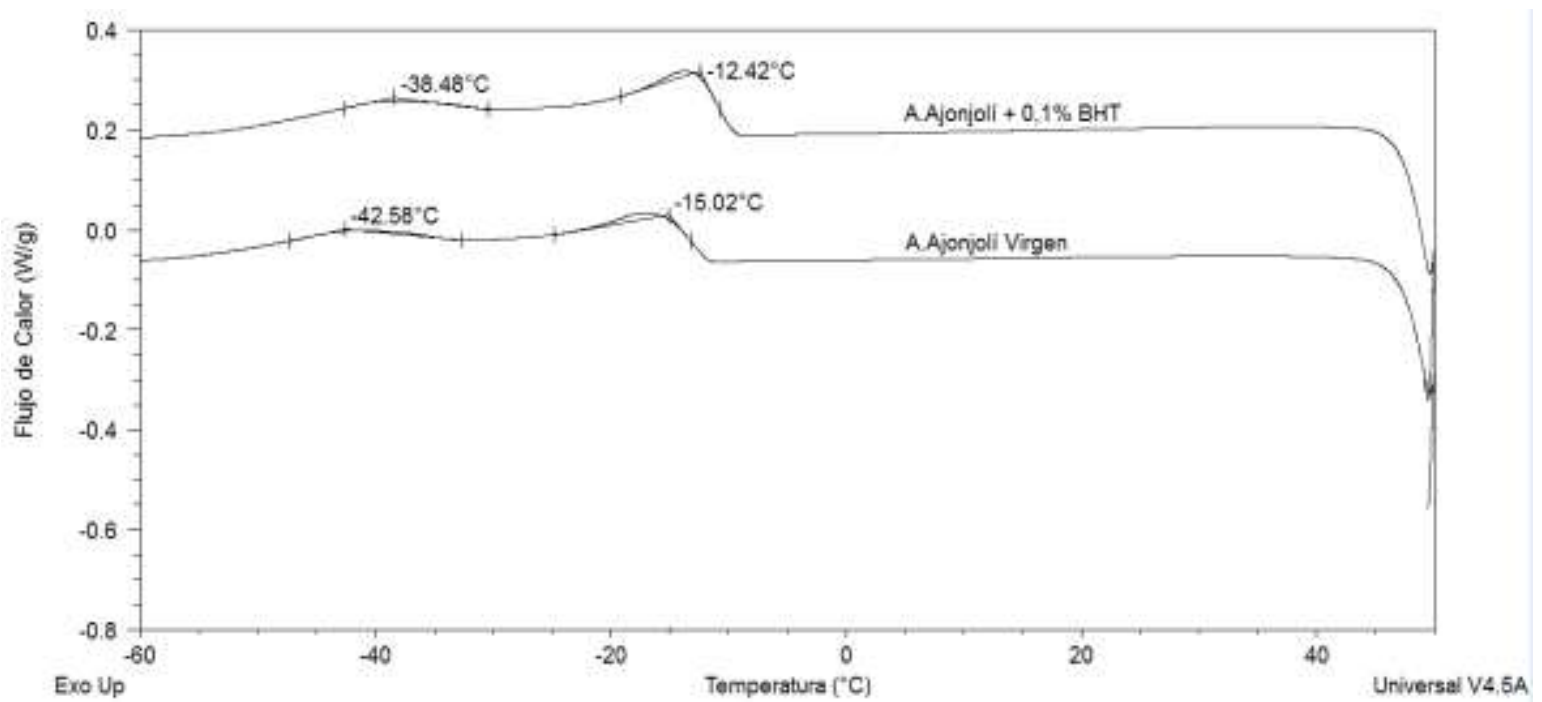

Fig. 5: Curvas de enfriamiento por DSC

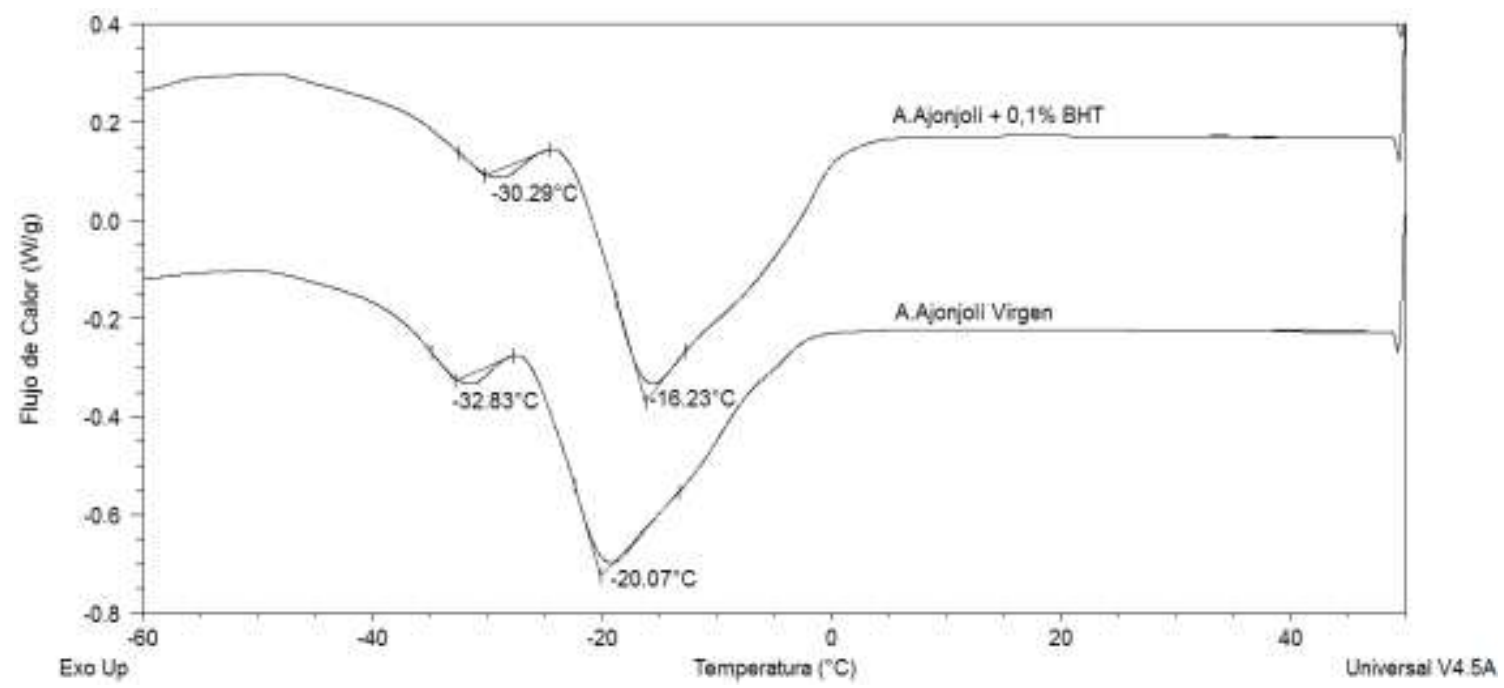

Fig. 6: Curvas de calentamiento por DSC

La adición de $0,1 \%$ de BHT afecta las temperaturas de cristalización y fusión, se requiere estudiar la fluidez a bajas temperaturas para verificar el efecto de la adición del mencionado antioxidante en el aceite de ajonjolí como posible base para la elaboración de lubricantes ecológicos.

El valor que determina la correlación existente entre la viscosidad del aceite y la temperatura es el índice de viscosidad; cuanto más alto es éste, menor es la variación de la viscosidad con la temperatura. El valor del índice de viscosidad del aceite de ajonjolí virgen fue 157,87, el de la mezcla con 0,1\% de BHT fue 159,49. No hay efecto del antioxidante sobre esta propiedad.

\section{CONCLUSIONES}

Se evaluó el poder antioxidante del BHT en aceite de ajonjolí extra virgen mediante análisis de estabilidad oxidativa por DSC. Se encontró que la concentración del antioxidante al $0,1 \%$ fue más eficiente con respecto a otras concentraciones, es importante mencionar que el BHT fue soluble en el aceite.

La adición de $0,1 \%$ de BHT disminuyó la degradación del aceite en la prueba tribológica de desgaste preventivo según los resultados de Valor de Peróxidos y tiempo de inducción a la oxidación por DSC, resultado predecible según el análisis previo de estabilidad oxidativa. A esta concentración el BHT puede disminuir ligeramente la fluidez del aceite de ajonjolí según las temperaturas obtenidas de los picos de cristalización y fusión por DSC. Se requiere en investigaciones futuras estudiar el efecto del BHT sobre la fluidez del aceite. Otra propiedad importante de los lubricantes como es el índice de viscosidad, no fue afectado por la adición de 0,1\% BHT al aceite de ajonjolí. 


\section{REFERENCIAS}

Adhvaryu, A., S.Z. Erhan y J.M. Perez, Wax Appearance Temperatures of Vegetable Oils Determined by Differential Scanning Calorimetry: Effect of Triacylglycerol Structure and Its Modification, Thermochimica Acta: 395, 191-200 (2002)

A.O.A.C. 965.33, Peroxide Value of Oils and Fats, (1990)

ASTM D445-12, Standard Test Method for Kinematic Viscosity of Transparent and Opaque Liquids (and Calculation of Dynamic Viscosity), 1-5, ASTM International, (2012)

ASTM D4172-94, Standard Test Method for Wear Preventive Characteristics of Lubricating Fluid (Four-Ball Method), 1-5, ASTM International, (2010)

Delgado, A.E. y W.A. Aperador, Estudio Comparativo del Poder Lubricante y Estabilidad Oxidativa entre el Aceite de Ajonjolí y Aceite Mineral 360, Información Tecnológica: 25 (4), 79 - 90 (2014)

Fox, N.J. y G.W. Stachowiak, Vegetable Oil-Based Lubricants - A Review of Oxidation, Tribology International: 40(7), 1035-1046 (2007)

Hemalatha, S. y Ghafoorunissa, Sesame Lignans Enhance the Thermal Stability of Edible Vegetable Oils, Food Chemistry: 105, 1076-1085 (2007)

Hernández, Carlos, Mieres, Alberto, Niño, Zulay y Pérez, Sergio, Efecto de la Refinación Física Sobre el Aceite de la Almendra del Corzo (Acrocomia aculeata), Información Tecnológica: 18(5), 59-68 (2007)

Igoe, Robert S., Dictionary of Food Ingredients, Fifth Edition, 21, Springer, New York (2011)

Jiménez, A., G. Beltrán, M.P. Aguilera y M. Uceda, Calorimetría Diferencial de Barrido. Influencia de la Composición del Aceite de Oliva Virgen en su Perfil Térmico, Grasas y Aceites: 58 (2), 122-129 (2007)

Lafont, Jennifer J., Durango, Luis C. y Aramendiz, Hermes, Estudio Químico del Aceite Obtenido a Partir de Siete Variedades de Soya (Glycinemax L.), Información Tecnológica: 25(2), 79-86 (2014)

Mannekote, J.K. y S.V. Kailas, The Effect of Oxidation on the Tribological Performance of Few Vegetable Oils, Journal of Materials Research and Technology: 1(2), 91-95 (2012)

Marteau, C., D. Favier, V. Nardello-Rataj y J. Aubry, Dramatic Solvent Effect on the Sinergy Between $\alpha$ tocopherol and BHT Antioxidants, Food Chemistry: 160, 190-195 (2014)

Martínez, N. e I. Jachmanián, Efecto de la Concentración de Diferentes Antioxidantes Sintéticos sobre la Estabilidad Oxidativa del Biodiesel, Facultad de Química, Universidad de la República, Uruguay (2013)

Navas, J. A., Optimización y Control de la Calidad y Estabilidad de Aceites y Productos de Fritura, Tesis de Doctorado, Dpto. de Nutrición y Bromatología, Universidad de Barcelona, Barcelona (2005)

Pardauil, J.J., L.K. Souza, F.A. Molfetta, J.R. Zamian, G.N. Rocha y C.E. da Costa, Determination of the Oxidative Stability by DSC of Vegetable Oils from the Amazonian Area, Bioresource Technology: 102 (10), 5873-5877 (2011)

Quinchia, L.A., M.A. Delgado, J.M. Franco, H.A. Spikes y C. Gallegos, Low-temperature Flow Behaviour of Vegetable Oil-based Lubricants, Industrial Crops and Products: 37 (1), 383-388 (2012).

Rizwanul, I.M. y otros 6 autores, Effect of Antioxidants on Oxidation Stability of Biodiesel Derived from Vegetable and Animal Based Feedstocks, Renewable and Sustainable Energy Reviews: 30, 356-370 (2014)

Rojano, B.A., Oxidación de Lípidos y Antioxidantes (en línea), 1997. http://www.bdigital.unal.edu.co/8413/1/6884161.1997.pdf. Acceso: 9 de Abril (2014)

San José, J., Sanz-Tejedor, M.A. y Arroyo, Y., Effect of Fatty Acid Composition in Vegetable Oils on Combustion Processes in an Emulsion Burner, Fuel Processing Technology: 130(2015), 20-30 (2014).

Sathwik, K.R., N. H. Jayadas y S. Kailas, Natural Oil-Based Lubricants, Green Energy and Technology: 287$328(2012)$

Tan, C.P. y Y.B. Che Man, Differential Scanning Calorimetric Analysis of Edible Oils: Comparison of Thermal Properties and Chemical Composition, Journal of the American Oil Chemists' Society: 77(2), 143-155 (2000)

Tan, C.P., Y.B. Che Man, J. Selamat y M.S.A. Yusoff, Comparative Studies of Oxidative Stability of Edible Oils by Differential Scanning Calorimetry and Oxidative Stability Index Methods, Food Chemistry: 76 (3), 385-389 (2002)

Tuner, H. y Korkmaz, M., Radiostability of Butylated Hydroxytoluene (BHT): An ESR Study, Nuclear Instruments and Methods in Physics Research B: 258, 388-394 (2007) 